\title{
Monostatic and Bistatic Low Frequency Ultrawideband SAR Imaging Experiment
}

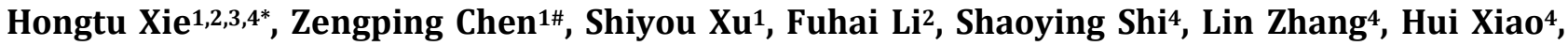 \\ Guangxue Wang4, Chao Xie ${ }^{4}$, Guoqian Wang ${ }^{*}$
}

${ }^{1}$ School of Electronics and Communication Engineering, Sun Yat-sen University, Shenzhen, China

${ }^{2}$ College of Electrical and Information Engineering, Hunan University, Changsha, China

${ }^{3}$ School of Electronic Information and Electrical Engineering, Shanghai Jiao Tong University, Shanghai, China

${ }^{4}$ Air Force Early Warning Academy, Wuhan, China

${ }^{5}$ Affiliated Hospital, Hunan Institute of Traditional Chinese Medicine, Changsha, China

Email: "xht20041623@163.com

How to cite this paper: Xie, H.T., Chen, Z.P., Xu, S.Y., Li, F.H., Shi, S.Y., Zhang, L., Xiao, H., Wang, G.X., Xie, C. and Wang, G.Q. (2018) Monostatic and Bistatic Low Frequency Ultrawideband SAR Imaging Experiment. Journal of Computer and Communications, 6, 67-76.

https://doi.org/10.4236/jcc.2018.611006

Received: April 9, 2018

Accepted: November 12, 2018

Published: November 19, 2018

\begin{abstract}
Low frequency ultrawideband (LF UWB) synthetic aperture radar (SAR) has lately become of a particular interest to SAR community. Monostatic and bistatic LF UWB SAR system has the well foliage penetrating capability, high-resolution imaging and providing the increased information. In 2015, a monostatic and bistatic LF UWB SAR imaging experiment was conducted. In this experiment, the monostatic and bistatic data were collected simultaneously by operating a moving vehicle-based radar in the SAR mode, in conjunction with a stationary ground-based receiver. The aim was to investigate the imaging property of the bistatic LF UWB SAR system. The one pulse per second (1 PPS) signal in combination with the global position system (GPS) disciplined $100 \mathrm{MHz}$ oscillator from the GPS receivers was used to implement the time and frequency synchronization in this SAR system. The bistatic SAR image was obtained by the subaperture spectrum-equilibrium method integrated with the fast factorized back projection (FFBP) algorithm. Bistatic experiment results are show to prove the validity of the bistatic LF UWB SAR imaging experiment.
\end{abstract}

\section{Keywords}

Bistatic Synthetic Aperture Radar, Low Frequency Ultrawideband, Imaging Experiment

\section{Introduction}

Synthetic aperture radar (SAR) plays a significant role in the remote sensing ap${ }^{*}$ Hongtu Xie and Guoqian Wang are co-first authors. 
plications, so it has attracted the wider attention in both military and civilian fields [1]-[6]. Based on the monostatic SAR system, several bistatic SAR systems were built by several countries, and the imaging experiments of the monostatic and bistatic SAR were conducted [6] [7] [8] [9]. Compared with the monostatic SAR case, the bistatic SAR system has several different properties [10]. The bistatic SAR can satisfy the different applications, since it has more complicated configuration and it can be designed flexibly. For the same scene, the bistatic SAR system has the greater anti-jamming ability than the monostatic SAR. If the bistatic SAR system includes more than one receivers or transmitters, the scene can be illuminated at the different angles, which can acquire the increased information of the targets. The one-stationary bistatic SAR is a special bistatic SAR system [11] [12] [13], which has a moving transmitter (receiver) and a stationary transmitter (receiver). One-stationary bistatic SAR system not only inherits the advantages of the general bistatic SAR system, but also is easy to build and implement imaging, thus it has attracted the wide attention.

Low frequency $(<1 \mathrm{GHz})$ ultrawideband (LFUWB) SAR system works in the LF signal with a big fractional bandwidth and has the wide antenna beam width with a big integration angle, so it has the well foliage penetrating ability and high-resolution imaging, which can detect the concealed targets under the foliage [14] [15] [16] [17]. Hence, it is a significant advantage that the monostatic and bistatic SAR systems work using the LFUWB transmitted signal. Some countries have conducted the imaging experiment of the monostatic and bistatic LF UWB SAR, and the excellent results were obtained [18] [19] [20] [21] [22].

Compared with the monostatic LF UWB SAR system, the bistatic LF UWB SAR system poses the various challenges in the imaging experiment, such as the synchronization between the transmitter and receiver, precise position measurement of radars, polar imetric calibration, collection geometry planning, data processing, etc. Most important challenge is a successful operation of the bistatic LF UWB SAR imaging experiment.

Based on the monostatic LF UWB SAR, we conducted a programme of the upgrades to add a bistatic collectionability. This work was completed and the imaging experiment of the monostatic and bistatic LF UWB SAR was conducted in 2015, and the monostatic and bistatic data were collected simultaneously by operating a moving vehicle-based radar in a SAR mode, in conjunction with a stationary ground-based receiver.

In this paper, the monostatic and bistatic LF UWB SAR system is given in Section 2, mainly focusing on the bistatic collection ability. The imaging experiment is discussed in Section 3. The imaging results are presented in Section 4. Section 5 gives a conclusion.

\section{Development of Monostatic and Bistatic LF UWB SAR System}

Monostatic LF UWB SAR system was developed by the National University of Defense Technology in 2008 [16], which operated at the LF signal with the frac- 
tional bandwidth about 0.25 . In 2011, a programme of the hardware and software upgrades to add the bistatic collection ability to this monostatic LF UWB SAR system was commenced. In 2015, the imaging experiment has been conducted in campus in Changsha City, Hunan Province, P.R. China. The vehicle-based LF UWB SAR system transmits the chirp signal and receives the scattered signal of the scene, simultaneity the ground-based LF UWB receiver illuminates the same region and receives the scattered signal of the scene.

\subsection{Overview}

Vehicle-based SAR system contains the transmitting/receiving antennas, global positioning system (GPS) antenna, transmitter/receiver, GPS motion compensation module, processor, etc. To void the antenna's coupling and interfere for the near-field imaging, the caliber coupling multilayer patch antennas for transmitting the radar signals and receiving the scattered signals are mounted in the vehicle-based SAR system separately. Transmitting and receiving antennas have a $3 \mathrm{~dB}$ azimuth beam width from approximately $30^{\circ}$ to $55^{\circ}$ and a $3 \mathrm{~dB}$ elevation beam width from approximately $70^{\circ}$ to $90^{\circ}$. Transmitter/receiver has the center controller, GPS synchronization module, frequency synthesizer, wave generator, etc. One pulse per second (1 PPS) signal from the GPS receiver is transferred into the center controller to control the timing reference of the vehicle-based SAR system. GPS synchronization module provides the coherence frequency reference for the vehicle-based SAR system, and then it is used to drive a frequency synthesizer to produce various clock signals for the different parts of the vehicle-based SAR system. Under the control of the center controller, the wave generator generates the chirp signal. It then is modulated into the radio frequency signal, attenuated by the adjustable attenuator and amplified by the power amplifier. At last, it is transmitted by the transmitting antenna. Simultaneity, the receiving antenna receives the scene scattered signal. Then, it is transferred into the low noise amplifier, down-converted into the intermediate frequency signal, filtered and amplified by the power amplifier, and demodulated into the in-phase and quadrature signals. Finally, in-phase and quadrature signals are sampled by a high-speed sampler, converted by the analogue-to-digital converter, and recorded on the solid state electronic array. Vehicle-based SAR system position can be obtained by the GPS motion compensation module and recorded on the solid state electronic array.

Ground-based receiving system has the receiving antenna, receiver, GPS antenna, processor, etc. The same caliber coupling multilayer patch antenna is used to receive the scene scattered signal. The ground-based receiver system has the similar function to the receiving part of the vehicle-based SAR system, which only receives the scattered signal, processed by the processor and recorded on the electronic array.

\subsection{Synchronization}

Synchronization of the monostatic and bistatic LF UWB SAR system mainly in- 
cludes the space, time and frequency synchronization. Firstly, the space synchronization between the vehicle-based SAR system and ground-based receiving system can be easily achieved, i.e., the ground-based receiving antenna illuminates the invariable region, and the vehicle-based transmitting antenna illuminates the same region in the spotlight mode.

Secondly, to achieve time and frequency synchronization between vehiclebased SAR system and ground-based receiving system, the GPS receivers have been used as the reference clock and trigger generators. 1 PPS signal from the GPS receivers is an accessible trigger useful for the bistatic requirement, which can solve the timing synchronization problem. Some GPS receivers offer a 100 $\mathrm{MHz}$ reference clock synchronized to the 1 PPS signal. Such a type of the GPS disciplined oscillator is used as the system reference clock and is a solution for the frequency synchronization issue. Voltage-controlled oscillator (VCO) generates the standard sine signal. After the amplification and frequency conversion, it is transferred to a phase detector, and it is phase detected by the 1 PPS signal. The phase error from the phase detector is used to discipline the VCO, and the output signal from them in turn stabilizes the VCO by the digital phase-locked loop. VCO produces the coherent reference signals in two systems, and are used to drive a frequency synthesizer to produce various signals in each system. Thus, 1 PPS signal in combination with the GPS disciplined $100 \mathrm{MHz}$ oscillator allow the vehicle-based SAR and ground-based receiving systems to become coherent and synchronized, which could real-timing satisfy the time and frequency synchronization.

\section{Monostatic and Bistatic LF UWB SAR Imaging Experiment}

\subsection{Experimental Test}

The bistatic collection ability of the vehicle-based LF UWB SAR system was exercised in 2015. Monostatic and bistatic LF UWB SAR systems were performed well, simultaneously producing a set of the monostatic and bistatic scattered signal of the scene.

Figure 1 shows the monostatic and bistatic LF UWB SAR system. Ground-based receiving system was placed on the step in the west of the square, with the receiving antenna mounted on the roof of a tripod. Vehicle-based SAR system was integrated on board a vehicle, and transmitting and receiving antennas was mounted at the right front and right rear of the vehicle's top. The dashed line is its ideal track, and the solid curve is its actual track. Vehicle-based transmitting antenna illuminates the scene including several targets, and the vehicle-based and ground-based receiving antennas receive the monostatic and bistatic scattered signals of the scene. Parameters of the monostatic and bistatic LF UWB SAR is listed in Table 1. The vehicle moves parallel to the $\mathrm{X}$-axis with an average speed $12.8 \mathrm{~km} / \mathrm{h}$, and the average height of the vehicle-based antenna is $4 \mathrm{~m}$. The position of the ground-based antenna is about $(-54,0,6) \mathrm{m}$, and the initial position of the vehicle-based antennas are about $(-38,26,4) \mathrm{m}$ and $(-42,26,4) \mathrm{m}$ at zeros time. 


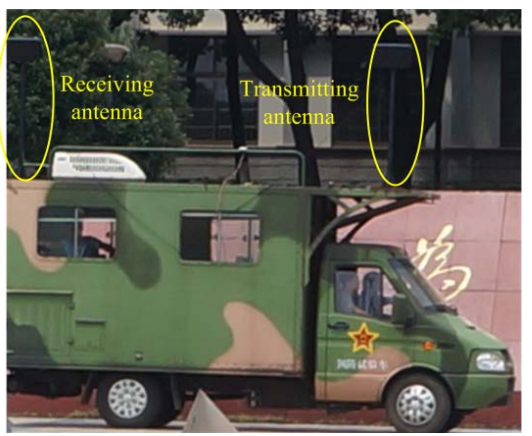

(a)

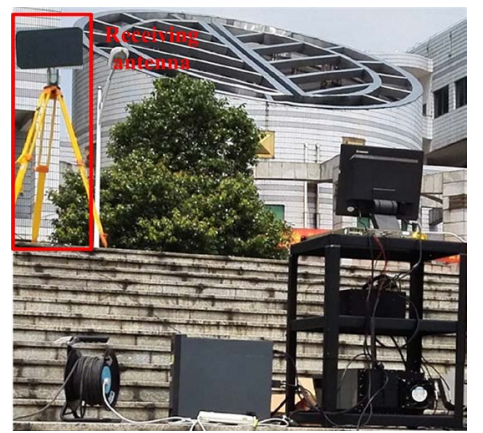

(c)

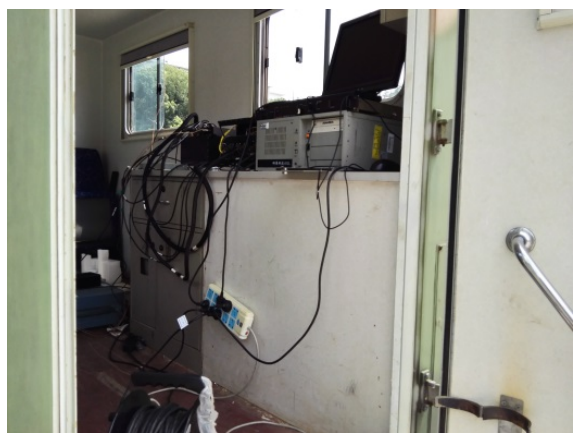

(b)

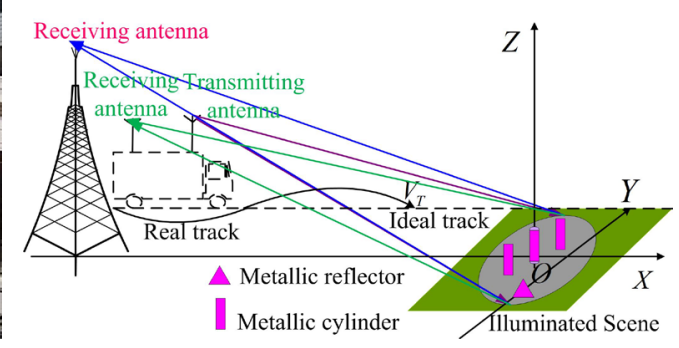

(d)

Figure 1. Monostatic and bistatic LF UWB SAR system. (a) Vehicle-based LF UWB SAR system; (b) Carriage installation viewed from outside; (c) Ground-based LF UWB receiving system; (d) Imaging geometry.

Table 1. Experiment parameters of the monostatic and bistatic LF UWB SAR.

\begin{tabular}{cccc}
\hline Parameters & Values & Parameters & Values \\
\hline Signal frequency & P-band & Sampling frequency & $220 \mathrm{MHz}$ \\
$\begin{array}{c}\text { Pulse repetition frequency } \\
\begin{array}{c}\text { Ground-based receiving } \\
\text { system position }\end{array}\end{array}$ & $500 \mathrm{~Hz}$ & Pulse duration & $100 \mathrm{~ns}$ \\
Vehicle ideal speed & $12.8 \mathrm{~km} / \mathrm{h}$ & Vehicle-based antenna ideal altitude & $4 \mathrm{~m}$ \\
\hline
\end{tabular}

As the scene being imaged must lie in the line-of-sight of the ground-based receiving antenna, and therefore the constrained operation of the monostatic and bistatic SAR system to the imaging of the test-sites within only a few decameters from the tripod. To collect the scattered data over a range of the incidence angle, the distance of the scene center is varied: the typical distance ranged from $35 \mathrm{~m}$ to $55 \mathrm{~m}$, which produces the incidence angle of the vehicle-based antennas from $80.3^{\circ}$ to $80.8^{\circ}$, and then the incidence angle of ground-based antenna from $85.91^{\circ}$ to $86.9^{\circ}$. Finally, one data site was selected for the imaging: the scene was a flat area, which has the size about $50 \mathrm{~m}$ in the $\mathrm{X}$ axis direction and $35 \mathrm{~m}$ in the $\mathrm{Y}$ axis direction. The position of the scene center is $(0,0,0) \mathrm{m}$. There are various scattering targets, mainly including one metallic trihedral reflector and three metallic cylinders, which were deployed at the test-site prior to this imaging experiment. Position of the metallic trihedral reflector is about (7.5, 
$-17.5,0) \mathrm{m}$, and the positions of the metallic cylinders are about $(5.5,5.5,0) \mathrm{m}$, $(0,0,0) \mathrm{m}$, and $(-5.5,-5.5,0) \mathrm{m}$.

\subsection{Image Formation}

The bistatic LF UWB SAR system is subject to some difficulties in the image formation, i.e., huge amount of the scattered signals, large spatial-variance, serious range-azimuth coupling and complicated motion errors, as well as the serious radio frequency interfere (RFI), which makes the image formation more complicated. The accurate disposal of these problems is an intractable issue for the frequency domain algorithms, but it can be precisely managed using the time domain back projection (BP) algorithm [23]. To reduce its high computation, fast implementation of the BP algorithm has been applied for the bistatic SAR image formation, i.e., fast factorized BP (FFBP) algorithm [24]. Application of the FFBP algorithm to process the bistatic narrowband SAR data was given in [24], which gave a basic principle of the bistatic FFBP algorithm, but did not give its implementation. The bistatic FFBP algorithm was applied for the space borne-airborne bistatic SAR imaging [25], and it represented the subimages in the elliptical polar grids. However, the sampling requirement for elliptical polar grids was derived not only for the linear track bistatic case, but also for the preferred case of radars with a higher angular speed. Another FFBP algorithm in the Cartesian coordinate for the bistatic UWB SAR data processing was given in [26]. The sampling requirement of the beams wasn't given, and the requirements for splitting the subaperture and subimage were derived only for the linear track case. A FFBP algorithm was given for the bistatic LF UWB SAR with a non-linear track [15], which can be used for this bistatic SAR image formation. And, the monostatic LF UWB SAR data can be processed by the monostatic FFBP algorithm.

In addition, there may be very strong RFI saturated by the receiving antenna working in the LF UWB signal. The influence of the RFI signals may be very significant because their power is much higher than the reflected power of the LF UWB SAR data. In order to remove its influence, a subaperture spectrum equilibrium method integrated with a FFBP algorithm is used in the LF UWB SAR image formation.

Before the RFI signal is suppressed, the monostatic and bistatic LF UWB SAR data (including the GPS data) must be preprocessed. First, GPS data should be transferred into the Cartesian coordinate system to obtain the local position of the vehicle-based antennas. Second, the scene scattered signals should be down sampled and filtered at the slow time. Finally, the scattered signals are range compressed by a matching filter function, and then the RFI in the scattered signal is suppressed by the subaperture-based spectrum equilibrium method. In this image formation, the direct-path signal from the vehicle-based transmitting antenna to the vehicle-based and ground-based receiving antennas is used to correct the time error of the scattered signal caused by the uncertain time delay. 


\section{Bistatic Experiment Results}

Figure 2 gives the bistatic scattered signal in time domain and frequency domain. Form Figure 2(a), the real (blue line) and imaginary components (red line) of the bistatic scattered signal are complicated due to the bistatic geometry. Form Figure 2(b), it is seen that the scattered signal of the targets in the frequency domain, since the targets are deployed at the flat and open square, and the power of their scattered signal is strong.

Figure 3 shows the imaging result of the bistatic LF UWB SAR scattered signal, and it is shown that the scene is well reconstructed by the FFBP algorithm integrated with the subaperture spectrum equilibrium method. Besides, it can be seen only a small part of the bistatic SAR image appears well illuminated. Since the ground-based receiving antenna results in the narrow antenna footprint, which may produce the rapid roll-off of brightness towards the lateral edges of the bistatic image. First, it is seen that the metallic targets (the trihedral reflector and cylinders) are well focused, and the UWB features (i.e., orthogonal and nonorthogonal sidelobes) can be clearly observed. Second, it can be found that the relative scattered power of the metallic cylinder in the yellow ellipse will become strong compared with the metallic trihedral reflector in the red rectangle, due to the anisotropic characteristic of the metallic cylinders.

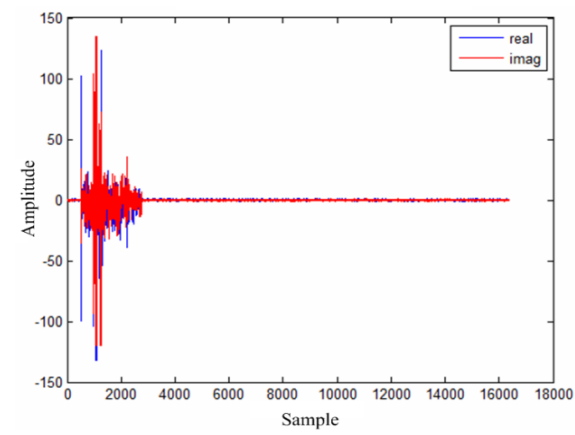

(a)

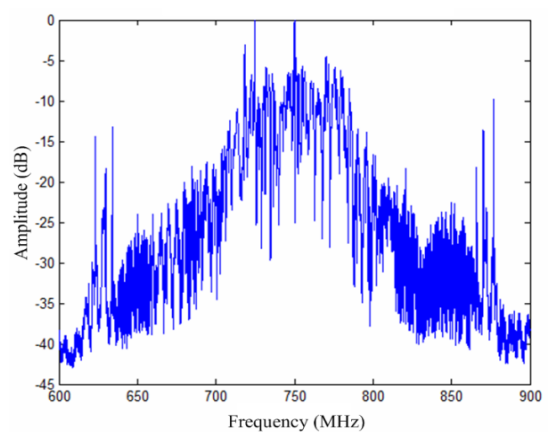

(b)

Figure 2. Bistatic scattered data of the illuminated scene. (a) Bistatic scattered data from one transmitted radar pulse, including the real (blue line) and imaginary component (red line); (b) Sum of the frequency spectrum of the monostatic scattered data from 100 radar pulses.

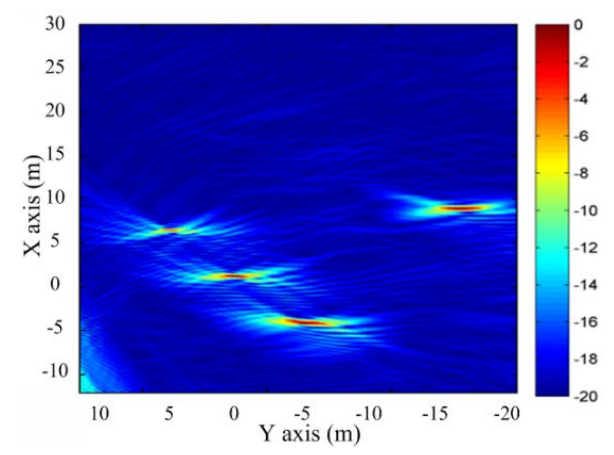

Figure 3. Imaging result of the bistatic LF UWB SAR scattered signal. 
To quantitatively evaluate the focusing quality of the bistatic SAR image in Figure 3, the resolutions of the focused metallic reflector in the $\mathrm{X}$ and $\mathrm{Y}$ axes has been measured. The measured resolutions of the focused metallic reflector in the $\mathrm{X}$ and $\mathrm{Y}$ axes are $0.37 \mathrm{~m}$ and $1.87 \mathrm{~m}$, respectively. The expected resolutions indirectly prove the validity of the proposed imaging method. Finally, for the sake of brevity, the reconstruction time of the scene by the proposed imaging method is about $386 \mathrm{~s}$. It can be concluded that the proposed imaging method can satisfy the bistatic LF UWB SAR imaging in reality.

\section{Conclusion}

The bistatic collectionability was added to the monosatic LF UWB SAR system: this involved the construction of the ground-based receiver to operate in conjunction with the existing vehicle-based monostatic SAR system. Monostatic and bistatic data were collected by the monostatic and bistatic SAR system and then processed by the FFBP algorithm. Bistatic experiment results indicate that this system is able to obtain the focused bistatic LF UWB SAR image. Some bistatic airborne experiments will carried out with the future collections planned, in order to support various research activities.

\section{Acknowledgements}

This work was supported in part by the National Natural Science Foundation of China under Grants 61571447 and 51309232, in part by Domain Foundation of Equipment Advance Research under Grants 6140410301 and 6140410304, and in full by the Scientific Research Project of Education Department of Hunan under Grant 16C1233.

\section{Conflicts of Interest}

The authors declare no conflicts of interest regarding the publication of this paper.

\section{References}

[1] Cumming, I. and Wong, F. (2005) Digital Processing of Synthetic Aperture Radar Data: Algorithm and Implementation. Artech House, Norwood.

[2] Jakowatz, C., Wahl, D., Eichel, P., Ghiglia, D. and Thompson, P. (2012) Spotlight-Mode Synthetic Aperture Radar: A Signal Processing Approach. Springer, New York.

[3] An, D., Huang, X., Jin, T. and Zhou, Z. (2012) Extended Nonlinear Chirp Scaling Algorithm for High-Resolution Highly Squint SAR Data Focusing. IEEE Transactions on Geoscience and Remote Sensing, 50, 3761-3773. https://doi.org/10.1109/TGRS.2012.2183606

[4] An, D., Huang, X., Jin, T. and Zhou, Z. (2012) Extended Two-Step Focusing Approach for Squinted Spotlight SAR Imaging. IEEE Transactions on Geoscience and Remote Sensing, 50, 2889-2900. https://doi.org/10.1109/TGRS.2011.2174460

[5] Xie, H., An, D., Huang, X. and Zhou, Z. (2016) Spatial Resolution Analysis of Low 
Frequency Ultrawidebeam-Ultrawideband Synthetic Aperture Radar Based on Wavenumber Domain Support of Echo Data. Journal of Applied Remote Sensing, 9, 095033-1-20.

[6] Hellsten, H., Sahlin, S. and Dammert, P. (2014) Polarimetric Subsurface SAR Imaging Outcome of Theoretical Development and CARABAS III Tests. Proceedings of International Radar Conference, Lille, 13-17 October 2014, 1-8.

[7] Walterscheid, I., et al. (2010) Bistatic SAR Experiments with PAMIR and TerraSAR-X-Setup, Processing, and Image Results. IEEE Transactions on Geoscience and Remote Sensing, 48, 3268-3279. https://doi.org/10.1109/TGRS.2010.2043952

[8] Rodriguez-Cassola, M., et al. (2012) First Bistatic Spaceborne SAR Experiments with TanDEM-X. IEEE Geoscience Remote Sensing Letter, 9, 33-37. https://doi.org/10.1109/LGRS.2011.2158984

[9] Baqué, R., et al. (2010) LORAMbis A Bistatic VHF/UHF SAR Experiment for FOPEN. Proceedings of IEEE Radar Conference, Washington DC, 10-14 May 2010, 832-837. https://doi.org/10.1109/RADAR.2010.5494506

[10] Xie, H., An, D., Huang, X. and Zhou, Z. (2015) Fast Time-Domain Imaging in Elliptical Polar Coordinate for General Bistatic VHF/UHF Ultra-Wideband SAR with Arbitrary Motion. IEEE Journal of Selected Topics in Applied Earth Observations and Remote Sensing, 8, 879-895. https://doi.org/10.1109/JSTARS.2014.2347413

[11] Xie, H., An, D., Huang, X. and Zhou, Z. (2016) Efficient Raw Signal Generation Based on Equivalent Scatterer and Subaperture Processing for One-Stationary Bistatic SAR including Motion Errors. IEEE Transactions on Geoscience and Remote Sensing, 54, 3360-3377. https://doi.org/10.1109/TGRS.2016.2516046

[12] Xie, H., Shi, S., Xiao, H., Xie, C., Wang, F. and Fang, Q. (2016) Efficient Time-Domain Imaging Processing for One-stationary Bistatic Forward-Looking SAR including Motion Error. Sensors, 16, 1-27. https://doi.org/10.3390/s16111907

[13] Xie, H., An, D., Huang, X. and Zhou, Z. (2015) Research on Spatial Resolution of One-Stationary Bistatic Ultrahigh Frequency Ultrawidebeam-Ultrawideband SAR Based on Scattering Target Wavenumber Domain Support. IEEE Journal of Selected Topics in Applied Earth Observations and Remote Sensing, 8, 1782-1798. https://doi.org/10.1109/JSTARS.2015.2414412

[14] Davis, M. (2011) Foliage Penetration Radar: Detection and Characterization of Objects under Trees. Scitech, New York.

[15] Xie, H., An, D., Huang, X., Li, X. and Zhou, Z. (2014) Fast Factorised Backprojection Algorithm in Elliptical Polar Coordinate for One-Stationary Bistatic Very High Frequency/Ultra High Frequency Ultra-Wideband Synthetic Aperture Radar with Arbitrary Motion. IET Radar, Sonar and Navigation, 8, 946-956. https://doi.org/10.1049/iet-rsn.2012.0350

[16] An, D., Li, Y., Huang, X., Li, X. and Zhou, Z. (2014) Performance Evaluation of Frequency-Domain Algorithms for Chirped Low Frequency UWB SAR Data Processing. IEEE Journal of Selected Topics in Applied Earth Observations and Remote Sensing, 7, 678-690. https://doi.org/10.1109/JSTARS.2013.2265272

[17] An, D., Chen, L. and Huang, X. (2015) Modified Nonlinear Chirp Scaling Algorithm for One-Stationary Bistatic Low Frequency Ultrawideband Synthetic Aperture Radar Imaging. Journal of Applied Remote Sensing, 9, 095056-1-10. https://doi.org/10.1117/1.JRS.9.095056

[18] Rasmusson, J., et al. (2007) Bistatic VHF and UHF SAR for Urban Environments. Proceedings of SPIE Defence \& Security Symposium: SAR Techniques, Radar Sensor Technology XI, Orlando, 9-13 April 2007, 1-12. 
[19] Henke, D., Barmettler, A. and Meier, E. (2009) Bistatic Experiment with the UWBCARABAS Sensor-First Results and Prospects of Future Applications. Proceedings of IEEE International Geoscience and Remote Sensing Symposium, Capetown, July 2009, 234-237.

[20] Ulander, L., et al. (2010) Signal-to-Clutter Ratio Enhancement in Bistatic VHFBand SAR Images of Truck Vehicles in Forested and Urban Terrain. IET Radar, Sonar and Navigation, 4, 438-448. https://doi.org/10.1049/iet-rsn.2009.0039

[21] Ulander, L., Frölind, P., Gustavsson, A., Jonsson, T., Ragnarsson, R. and Stenström, G. (2014) Development of CARINA Bistatic VHF-Band SAR. Proceedings of International Radar Conference, Lille, 13-17 October 2014, 601-606. https://doi.org/10.1109/RADAR.2014.7060354

[22] Ulander, L., Frölind, P., Gustavsson, A., Ragnarsson, R. and Stenström, G. (2015) VHF/UHF Bistatic and Passive SAR Ground Imaging. Proceedings of IEEE Radar Conference, Arlington, 10-15 May 2015, 669-673.

https://doi.org/10.1109/RADAR.2015.7131080

[23] Xie, H., et al. (2017) Fast Factorized Backprojection Algorithm for One-Stationary Bistatic Spotlight Circular SAR Image Formation. IEEE Journal of Selected Topics in Applied Earth Observations and Remote Sensing, 10, 1494-1510. https://doi.org/10.1109/JSTARS.2016.2639580

[24] Ulander, L., Frölind, P., Gustavsson, A., Murdin, D. and Stenström, G. (2010) Fast Factorized Back-Projection for Bistatic SAR Processing. Proceedings of European Conference on Synthetic Aperture Radar, Aachen, 7-10 June 2010, 1-14.

[25] Rodriguez-Cassola, M., Prats, P., Krieger, G. and Moreira, A. (2011) Efficient Time-Domain Image Formation with Precise Topography Accommodation for General Bistatic SAR Configurations. IEEE Transactions on Aerospace and Electronic System, 47, 2949-2966. https://doi.org/10.1109/TAES.2011.6034676

[26] Vu, V., Sjögren, T. and Pettersson, M. (2013) Fast Time-Domain Algorithms for UWB Bistatic SAR Processing. IEEE Transactions on Aerospace and Electronic System, 49, 1982-1994. https://doi.org/10.1109/TAES.2013.6558032 